\title{
Perfil fisiometabólico de perros Pastor Belga Malinois y Pastor Holandés de alto rendimiento en respuesta a una sesión de entrenamiento para Ring Francés
}

\author{
Physiometabolic profile of Belgian Malinois Shepherd and Dutch Shepherd dogs \\ in response to a training session for French Ring \\ M Mendoza-Lara ${ }^{a}$, H Orozco-Gregorio ${ }^{a}$, R Ramírez-Necoechea ${ }^{a}, S$ Caballero-Chacón ${ }^{b}$, \\ R Hernández-González ${ }^{c}$, M Becerril-Herrera ${ }^{\mathrm{d}}$, P Mora-Medina ${ }^{\mathrm{e}}$, D Mota-Rojas ${ }^{\mathrm{a}}{ }^{*}$ \\ aDepartamento de Producción Agrícola y Animal, Universidad Autónoma Metropolitana, Xochimilco, México D.F., México. \\ bUniversidad Nacional Autónoma de México, FMVZ, México D.F., México. \\ ${ }^{c}$ Departamento de Investigación Experimental y Bioterio, Instituto Nacional de Ciencias Médicas y \\ Nutrición Salvador Zubirán, México D.F., México. \\ ${ }^{\mathrm{d} B e n e m e ́ r i t a ~ U n i v e r s i d a d ~ A u t o ́ n o m a ~ d e ~ P u e b l a, ~ T e z i u t l a ́ n, ~ P u e b l a, ~ M e ́ x i c o . ~}$ \\ eFacultad de Estudios Superiores de Cuautitlan, FESC, Universidad Nacional Autónoma de México, \\ México D.F., México.
}

\section{SUMMARY}

\begin{abstract}
This study evaluated the physiometabolic response to a training session for French Ring in two high performance breeds of dogs to. Twenty male and female Belgian Malinois Shepherd and Dutch Shepherd dogs were used. Animals aged 10 to 24 months and weighing between 30 and $35 \mathrm{~kg}$ (66-77 lbs) performed a French Ring routine for 20 minutes. Blood samples were taken at three moments: prior to training, immediately after exercise, and then $1 \mathrm{~h}$ later. All samples were drawn from the cephalic vein. Lactate, $\mathrm{pH}$, calcium, potassium and blood gas $\left(\mathrm{pCO}_{2}\right.$ and $\left.\mathrm{pO}_{2}\right)$ levels were determined using a critical blood variables analyzer. Heart rate and body temperature were also recorded. In both breeds, temperature, $\mathrm{pO}_{2}, \mathrm{pCO}_{2}$, lactate and $\mathrm{pH}$ levels increased $(\mathrm{P}<0.05)$ immediately after the training period, but decreased $1 \mathrm{~h}$ after exercise $(\mathrm{P}<0.05)$. The Belgian Malinois Shepherd dogs showed an increase $(\mathrm{P}<0.05)$ in cardiac frequency in the post-training period, with a later recovery $(\mathrm{P}<0.05)$ that tended towards initial values. The Dutch Shepherd breed displayed a decrease $(\mathrm{P}<0.05)$ in calcium levels after training that remained without change during recovery. In conclusion, these results provide biochemical and physiological data that will make possible to evaluate and estimate the potential for competitive performance of these breeds, improving the selection of these two dog breeds.
\end{abstract}

Palabras clave: ejercicio, perro, gasometría, variables fisiológicas.

Key words: exercise, dog, gasometry, physiological variables.

\section{INTRODUCCIÓN}

El esfuerzo físico representa la adaptación del organismo a condiciones de mayor actividad muscular, la cual puede conseguirse mediante diversos cambios en el organismo que van desde las estructuras celulares y procesos metabólicos, hasta el nivel integral de las actividades funcionales (Evans y col 1993, Grandjean y col 2003, Gómez y col 2004). Las adaptaciones cardiovasculares y respiratorias son un ejemplo de estos ajustes al esfuerzo físico y tienen por objetivo asegurar el aporte de oxígeno y de nutrimentos necesarios para la actividad muscular (Brooks y Gasser, 1980, Olmos y col 2008, Orozco y col 2010), así como permitir la eliminación de los desechos producidos por el metabolismo muscular (Grandjean y col 2003, Olmos y col 2010). Las sesiones de entrenamiento dan lugar

Aceptado: 05.04.2012.

* Calzada del Hueso 1100, UAM-X, Col. Villa Quietud, Coyoacán, 04960 D.F., México; dmota100@yahoo.com.mx a un proceso de estrés físico que requiere, entre otras adaptaciones, un incremento del gasto cardiaco (Evans y col 1993, Grandjean y col 2003, Gómez y col 2004), lo que permite el aumento de la capacidad de trabajo físico y rendimiento deportivo. Sin embargo, la adaptación y el desempeño durante estas actividades difieren entre individuos (Taylor y col 2008).

Para llevar a cabo la evaluación de la capacidad física, desde hace tiempo se han empleado algunas variables fisiológicas o bien metabolitos asociados a situaciones de estrés y a respuestas metabólicas al ejercicio (Rose y Bloomberg 1989), entre ellos la medición del ácido láctico en el músculo ejercitado y en los fluidos corporales (Warren y col 1999, Sánchez y col 2008). Asimismo, en caballos de carrera pura sangre se ha demostrado que la frecuencia cardiaca, el volumen total de glóbulos rojos y la concentración de hemoglobina pueden ser indicadores confiables en la evaluación de la aptitud física y del nivel de entrenamiento que presenta un caballo para realizar determinado ejercicio (Gómez y col 2004). 
Recientemente, mediante la determinación sanguínea de gases $\left(\mathrm{pO}_{2}\right.$ y $\left.\mathrm{pCO}_{2}\right)$, glucosa, electrólitos $\left(\mathrm{K}^{+}, \mathrm{Ca}^{++}\right.$, $\mathrm{Na}^{+}$) y lactato, se ha llevado a cabo la caracterización del perfil fisiometabólico en animales sometidos a procesos de estrés durante el nacimiento (Trujillo y col 2007, Orozco y col 2008, Sánchez y col 2009). El uso de estas técnicas ha permitido también la descripción de las modificaciones metabólicas y lesiones de canales durante el transporte y sacrificio en animales de abasto (Amtmann y col 2006, Mota-Rojas y col 2006, 2009, Carter y Gallo 2008, Werner y Gallo 2008, Tadich y col 2009, Becerril y col 2009, 2010) y de equinos sometidos a condiciones de ejercicio intenso (Taylor y col 1995). No obstante, en otras especies como los perros, aún no existen estudios en los que se haya caracterizado el perfil fisiometabólico de perros de alto rendimiento sometidos a sesiones de entrenamiento de Ring Francés, por lo que la selección de los ejemplares sigue siendo aproximativa, y con frecuencia todavía empírica (Grandjean y col 2003). Este tipo de determinaciones permitirían una selección más certera basada en la eficiencia de la respuesta a un proceso de estrés producto del ejercicio (Matwichuk y col 1999) como lo es el entrenamiento de perros de las razas Pastor Belga Malinois y Pastor Holandés durante la modalidad de Ring Francés. El presente trabajo tiene por objetivo la caracterización del perfil fisiometabólico de perros de las razas Pastor Belga Malinois y Pastor Holandés en respuesta a una sesión de entrenamiento de Ring Francés a través de la determinación sanguínea de gases, lactato, $\mathrm{pH}$, electrólitos, temperatura corporal y frecuencia cardiaca.

\section{MATERIAL Y MÉTODOS}

\section{LOCALIZACIÓN}

El estudio se realizó durante los meses de abril y mayo de 2009 en un centro de entrenamiento canino en la modalidad de Ring Francés, localizado al norte de Ciudad de México.

\section{ANIMALES}

El estudio incluyó 14 perros machos y 6 hembras de los cuales 8 fueron de la raza Pastor Holandés y 12 Pastor Belga Malinois con edades de 1,5 \pm 0,7 años, y con un peso de $33 \pm 2,5 \mathrm{~kg}$.

Para el estudio sólo fueron seleccionados perros clínicamente sanos.

\section{EVALUACIONES}

De cada perro se registraron la edad, el peso y sexo. Para determinar los cambios fisiológicos en respuesta a la sesión de entrenamiento, todas las mediciones de las variables fisiometabólicas se realizaron antes e inmediatamente después de la sesión de entrenamiento (preentrenamiento y postentrenamiento). Las variables evaluadas inmediatamente después del entrenamiento se obtuvieron $10 \mathrm{~s}$ después de concluir la sesión de ejercicios, mientras que las mediciones para evaluar la capacidad de recuperación de los perros se realizaron $1 \mathrm{~h}$ después de finalizado el ejercicio (Recuperación). La temperatura corporal (TC) fue obtenida de forma instantánea (1s) a través de un termómetro otal ${ }^{1}$ y la frecuencia cardiaca (FC) se determinó mediante un estetoscopio. Previa asepsia, las muestras sanguíneas se obtuvieron de la vena cefálica con una jeringa. El tiempo empleado en la obtención de la muestra fue de $<15 \mathrm{~s}$. Inmediatamente después del muestreo sanguíneo la sangre fue vertida en un tubo microcapilar (de $100 \mu \mathrm{l}$ ) con heparina de litio (sangre entera) y fue procesada por medio de un gasómetro de variables críticas sanguíneas ${ }^{2}$ ubicado en el centro de entrenamiento canino, el cual determinó los niveles sanguíneos de lactato $(\mathrm{mg} / \mathrm{dL}), \mathrm{pH}$, electrólitos $\left(\mathrm{Ca}^{++} \mathrm{mmol} / \mathrm{L}, \mathrm{K}^{+} \mathrm{mmol} / \mathrm{L}\right)$ y presiones parciales de $\mathrm{O}_{2}$ y $\mathrm{CO}_{2}\left[\mathrm{pO}_{2},(\mathrm{mmHg}), \mathrm{pCO}_{2}(\mathrm{mmHg})\right]$. Con la finalidad de evitar alteraciones previas en los animales que aún no eran evaluados, estos se mantuvieron lejos del sitio de entrenamiento.

\section{ENTRENAMIENTO DE LOS PERROS}

Para el estudio sólo se seleccionaron perros jóvenes con edades de 1,5 $\pm 0,7$. A estas edades todos los perros ya habían sido previamente entrenados en los ejercicios considerados en el estudio, y respondían a ellos de la misma forma independientemente de su edad. El entrenamiento se llevó a cabo por un entrenador acreditado por la Federación Canófila Mexicana (FCM).

La sesión de entrenamiento a la que fue sometido cada uno de los perros consistió de 20 minutos de una rutina de ejercicios descrita por el Reglamento de Ring Francés $(1997)^{3}$, la cual incluyó: Protección, defensa del manejador $\mathrm{y}$ ataque de frente con vara, lanzamiento y recobre del objeto lanzado, ataque en huida con vara del entrenador y ataque de frente con revólver, recobre de objeto a la vista y búsqueda, ladrido y conducción por parte del entrenador, recobre de un objeto fuera de la vista y ataque suspendido de frente con vara, cuidado y protección de objeto (canasta). La rutina fue la misma para cada uno de los animales durante este período de evaluación. Antes del inicio de la rutina todos los animales presentaban un ayuno completo de $10 \mathrm{~h}$.

\footnotetext{
ThermoScan Braun ${ }^{\circledR}$ (GMBH, Kronberg, Germany).

GEM Premier 3000, Instrumentation Laboratory Diagnostics USA/ Italy $^{\circledR}$.

3 Reglamento de Ring Francés. 1997. Disponible en: http://www. voraus.com/v2/modules/wfsection/html/RingFrancesMexico.pdf
} [Consultado el 23 de octubre de 2009]. 


\section{ANÁLISIS ESTADÍSTICO}

Para determinar la existencia de diferencias significativas entre los tres grupos de cada raza y entre razas tiempos de valoración en cada raza y entre razas, todas las variables (excepto el $\mathrm{pH}$ ) se analizaron mediante un análisis de varianza (ANOVA). La prueba de comparación múltiple de medias se realizó mediante el procedimiento de Tukey con un nivel de significancia de $\mathrm{P}<0,05$ (Steel y Torrie 1986). La variable $\mathrm{pH}$ se analizó con la prueba de Kruskal-Wallis para posteriormente realizar la comparación entre grupos mediante la prueba U de Mann-Whitney. Debido a que los niveles de $\mathrm{pH}$ corresponden a unidades logarítmicas, estos datos se expresan como medianas (rangos). Asimismo, para ambas razas se realizó un análisis de regresión lineal en el que se consideró al lactato como variable dependiente y la $\mathrm{pO}_{2}, \mathrm{pCO}_{2}, \mathrm{Ca}^{++}$y temperatura como variables independientes.

Todas las pruebas estadísticas se realizaron mediante el programa estadístico SAS para microcomputadoras (SAS ver. 9.0, 2004).
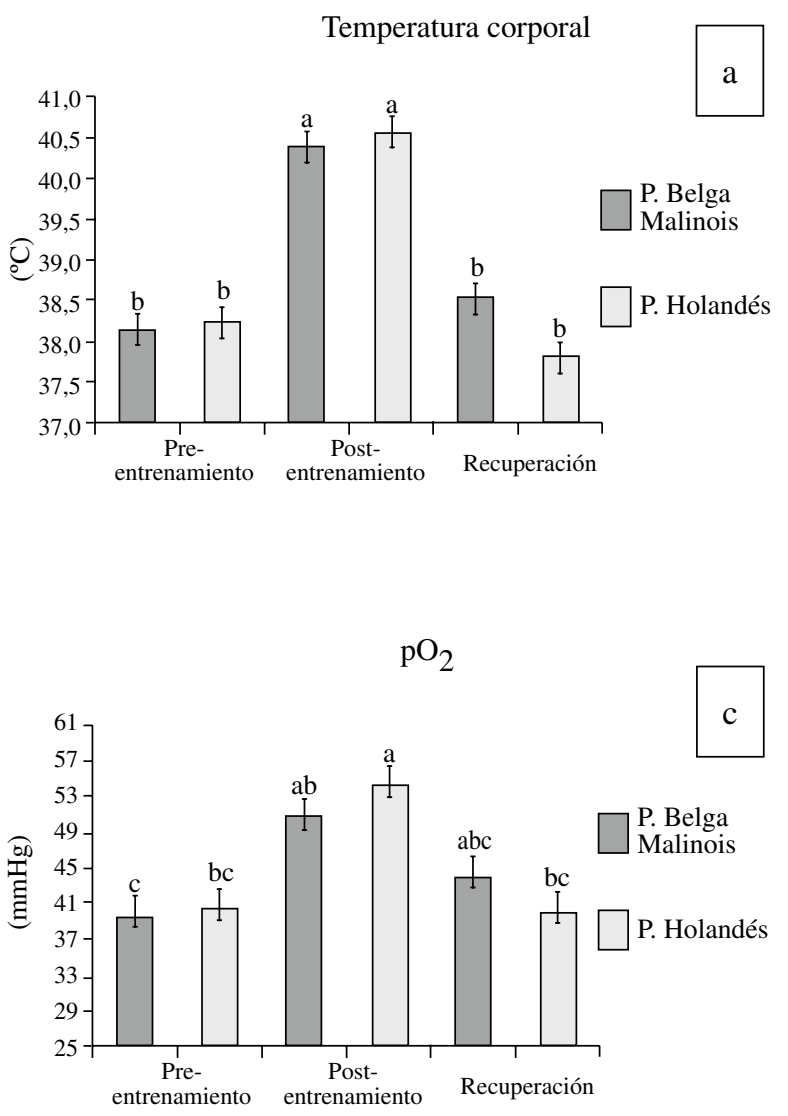

\section{RESULTADOS}

Los resultados de la TC, FC, $\mathrm{pO}_{2}$ y $\mathrm{pCO}_{2}$ se muestran en la figura 1. En ambas razas, la TC (Panel a) incrementó significativamente $(P<0,05)$ en el período postentrenamiento. Sin embargo, en el período de recuperación, la TC disminuyó significativamente $(\mathrm{P}<0,05)$ en las dos razas evaluadas.

La raza Pastor Belga Malinois presentó $8 \%$ más latidos por minuto $(\mathrm{P}<0,05)$ (FC, Panel b) en el período postentrenamiento en comparación con los niveles basales. Una hora después de finalizado el entrenamiento, los valores fueron similares a los iniciales $(\mathrm{P}<0,05)$. Sin embargo, estos cambios no se presentaron en la raza Pastor Holandés, en la que no se observaron cambios en ninguno de los tres tiempos de muestreo para esta variable.

En ambas razas las concentraciones sanguíneas de $\mathrm{pO}_{2}($ Panel c) incrementaron de manera significativa $(\mathrm{P}<0,05)$ inmediatamente después del entrenamiento con respecto a las concentraciones basales $(52,52$ vs $39,95 \mathrm{mmHg}$ ). No obstante, en el período de recuperación
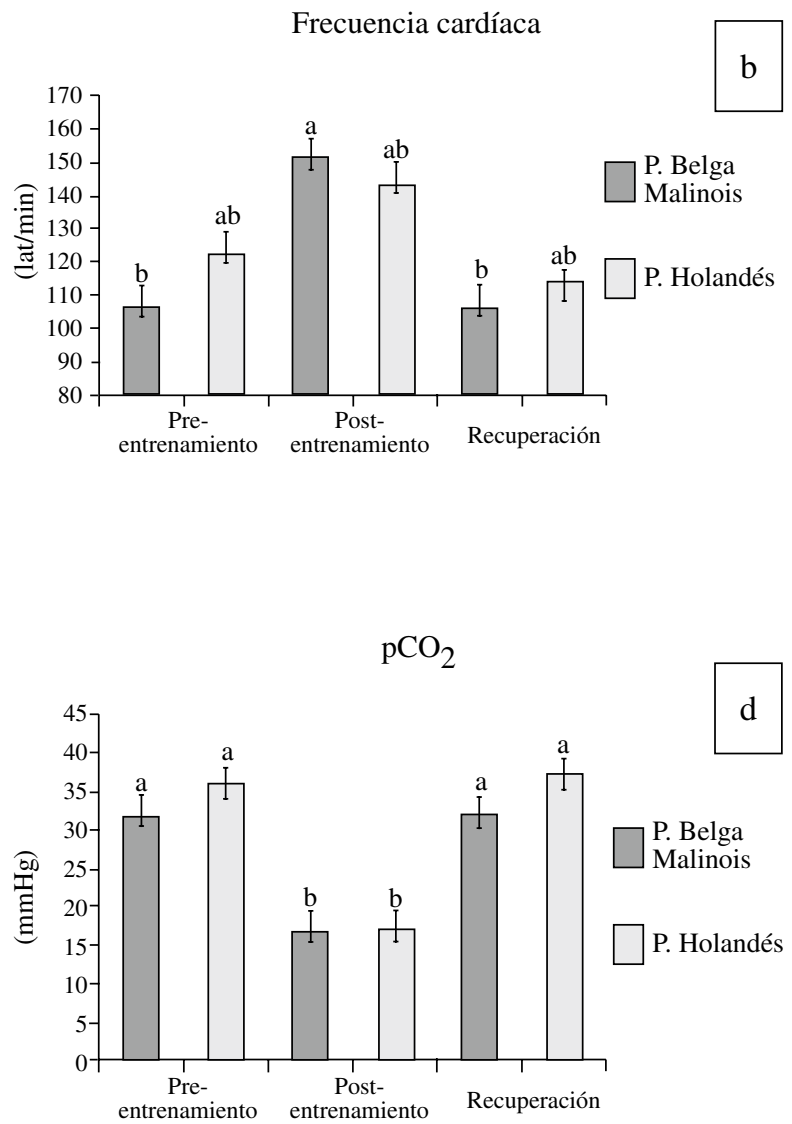

Figura 1. Efecto del entrenamiento en la TC, $\mathrm{FC}$ y concentraciones de $\mathrm{pO}_{2}$ y $\mathrm{pCO}_{2}$ en perros de las razas Pastor Belga Malinois y Pastor Holandés. Los datos se presentan como promedios \pm DE. Literales diferentes entre columnas $(\mathrm{a}, \mathrm{b}, \mathrm{c})$ indican diferencias significativas $(\mathrm{P}<0,05)$ entre los tres diferentes tiempos de evaluación comparados por raza y entre ambas razas.

Effect of training on $\mathrm{BC}, \mathrm{CF}$ and concentrations of $\mathrm{pO}_{2}$ and $\mathrm{pCO}_{2}$ in Belgian Malinois Shepherd and Dutch Shepherd dogs. Data are presented as averages $\pm \mathrm{SD}$. Different literals between columns $(\mathrm{a}, \mathrm{b}, \mathrm{c})$ indicate significant differences $(\mathrm{P}<0.05))$ among 3 different evaluation times compared by race and between races. 
sólo la raza Pastor Holandés logró regresar a los valores de inicio, mientras que en la raza P. Belga Malinois los niveles se mantuvieron sin cambios $1 \mathrm{~h}$ después de la etapa de entrenamiento. Con relación a la $\mathrm{pCO}_{2}($ Panel d), las concentraciones disminuyeron $(\mathrm{P}<0,05)$ en ambas razas durante el período postentrenamiento. En la etapa de recuperación, las dos razas incrementaron $(\mathrm{P}<0,05)$ sus concentraciones sanguíneas, observándose resultados similares a los basales.

En la figura 2 se presentan los resultados de las concentraciones de lactato, $\mathrm{pH}$ y electrólitos. En ambas razas las concentraciones de lactato (Panel a) incrementaron $(\mathrm{P}<0,05)$ aproximadamente $38,3 \mathrm{mg} / \mathrm{dl}$ después del entrenamiento en comparación con los niveles basales, sin embargo, después de $1 \mathrm{~h}$ las dos razas lograron restablecer sus concentraciones a niveles normales. Coincidentemente, el pH tuvo un comportamiento similar al lactato (figura 2, panel b).
Al comparar los resultados de $\mathrm{Ca}^{++}$y $\mathrm{K}^{+}$séricos entre razas (paneles c y d) no se aprecian diferencias significativas entre ellas en ninguno de los tres períodos de muestreo. Sin embargo, en la raza P. Holandés se presentó una disminución significativa $(\mathrm{P}<0,05)$ en las concentraciones de $\mathrm{Ca}^{++}$ después del entrenamiento respecto a las concentraciones basales (1,25 mmol/L vs 1,37 $\mathrm{mmol} / \mathrm{L})$. Esta situación no cambió durante la etapa de recuperación. En la raza $\mathrm{P}$. Belga Malinois los niveles sanguíneos de este indicador se mantuvieron estables durante todo el período de evaluación.

Como resultado del análisis de regresión lineal, en la raza P. Belga Malinois (cuadro 1) los niveles elevados de lactato postentrenamiento fueron relacionados significativamente $(\mathrm{P}<0,05)$ a niveles elevados de $\mathrm{pO}_{2}$ y bajos niveles de $\mathrm{pCO}_{2} \mathrm{y} \mathrm{Ca}^{++}$, mientras que en la raza P. Holandés, sólo se observó una relación significativa $(\mathrm{P}<0,05)$ entre niveles elevados de lactato y bajos niveles de $\mathrm{pCO}_{2}$ postentrenamiento (cuadro 2).
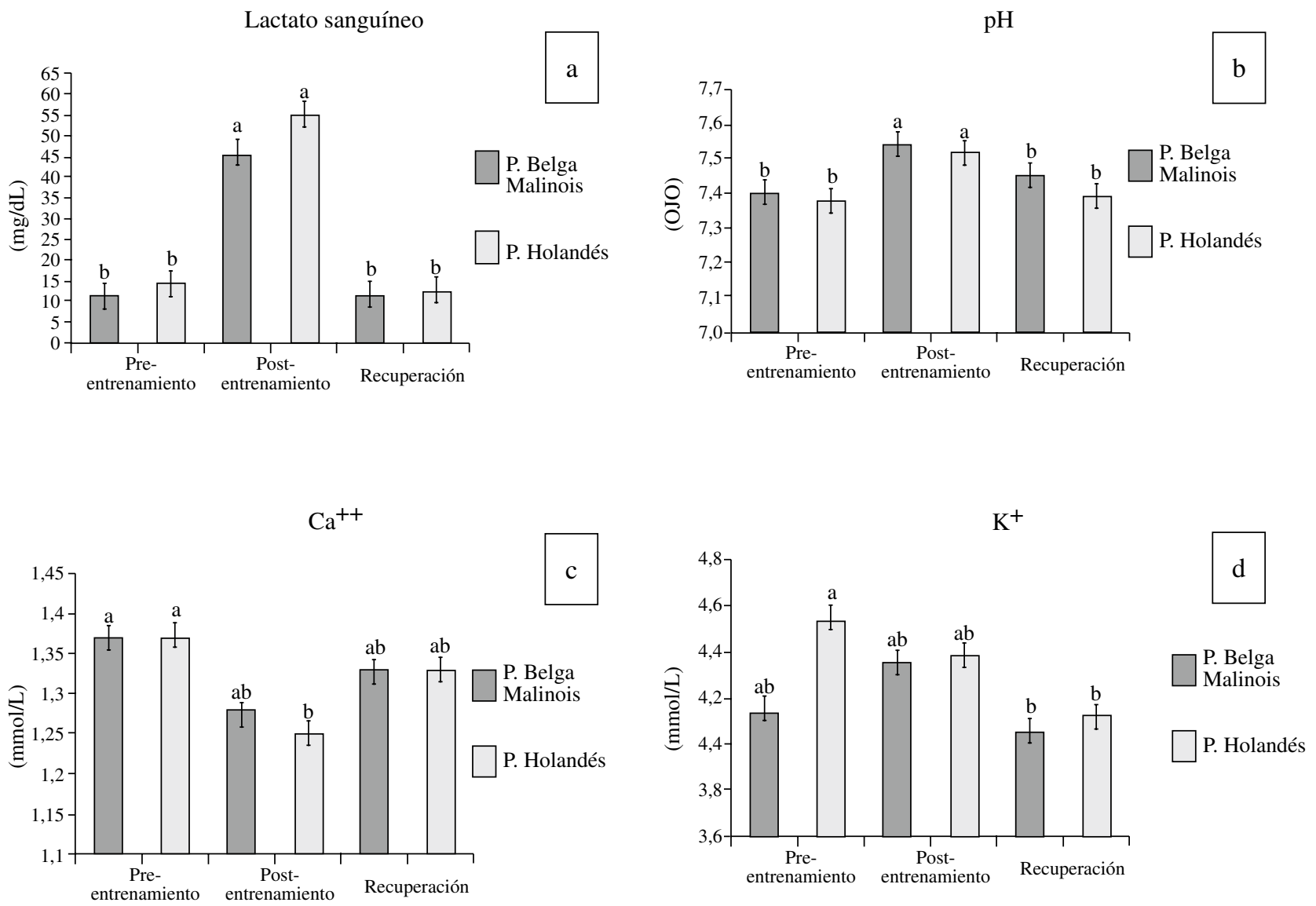

Figura 2. Efecto del entrenamiento en las concentraciones de lactato, $\mathrm{pH}, \mathrm{Ca}^{++}$y $\mathrm{K}^{+}$en perros de las razas Pastor Belga Malinois $\mathrm{y}$ Pastor Holandés. Los datos se presentan como promedios \pm DE. Literales diferentes entre columnas $(\mathrm{a}, \mathrm{b}, \mathrm{c})$ indican diferencias significativas $(\mathrm{P}<0,05)$ entre los tres diferentes tiempos de evaluación comparados por raza y entre ambas razas. La variable $\mathrm{pH}$ se expresa como mediana \pm rango y fue analizada mediante la prueba de Kruskal-Wallis $(\mathrm{P}<0,01)$.

Effect of training on concentrations of lactate, $\mathrm{pH}, \mathrm{Ca}^{++}$and $\mathrm{K}^{+}$in Belgian Malinois Shepherd and Dutch Shepherd dogs. Data are presented as averages $\pm \mathrm{DE}$. Different literals between columns $(\mathrm{a}, \mathrm{b}, \mathrm{c})$ indicate significant differences $(\mathrm{P}<0.05))$ among 3 different evaluation times compared by race and between races. The $\mathrm{pH}$ variable is expressed as mean \pm range and analyzed using the Kruskal-Wallis test $(\mathrm{P}<0.01)$. 
Cuadro 1. Ecuación de regresión de la variable lactato de la raza Pastor Belga Malinois.

Regression equation for the variable lactate in Belgian Malinois Shepherd dogs.

\begin{tabular}{ccccc}
\hline Variable dependiente $(\mathrm{y})$ & Variable independiente $(\mathrm{x})$ & Ecuación lineal $(\mathrm{y}=\mathrm{b}+\mathrm{mx})$ & Valor Correlación & $\mathrm{P}$ \\
\hline Lactato $(\mathrm{mg} / \mathrm{dL})$ & $\mathrm{pO}_{2}(\mathrm{mmHg})$ & $-33,49+\left(1,55 \mathrm{pO}_{2}\right)$ & 0,71 & 0,0004 \\
& $\mathrm{pCO}_{2}(\mathrm{mmHg})$ & $70,53+\left(-1,51 \mathrm{pCO}_{2}\right)$ & $-0,67$ & 0,0012 \\
& $\mathrm{Ca}^{++}(\mathrm{mmol} / \mathrm{L})$ & $180,59+(-105,08 \mathrm{Ca})$ & $-0,44$ & 0,0467 \\
& Temperatura $\left({ }^{\circ} \mathrm{C}\right)$ & $-138,82+(4,57$ Temperatura $)$ & 0,41 & 0,0785 \\
\hline
\end{tabular}

Cuadro 2. Ecuación de regresión de la variable lactato del Pastor Holandés. Regression equation for the variable lactate in Dutch Shepherd dogs.

\begin{tabular}{ccccc}
\hline Variable dependiente $(\mathrm{y})$ & Variable independiente $(\mathrm{x})$ & Ecuación lineal $(\mathrm{y}=\mathrm{b}+\mathrm{mx})$ & Valor Correlación & $\mathrm{P}$ \\
\hline Lactato $(\mathrm{mg} / \mathrm{dL})$ & $\mathrm{pO}_{2}(\mathrm{mmHg})$ & $1,18+\left(0,99 \mathrm{pO}_{2}\right)$ & 0,60 & 0,0377 \\
& $\mathrm{pCO}_{2}(\mathrm{mmHg})$ & $60,59+\left(-0,32 \mathrm{pCO}_{2}\right)$ & $-0,27$ & 0,3919 \\
& $\mathrm{Ca}^{++}(\mathrm{mmol} / \mathrm{L})$ & $135,05+\left(-63,66 \mathrm{Ca}^{++}\right)$ & $-0,33$ & 0,2857 \\
& Temperatura $\left({ }^{\circ} \mathrm{C}\right)$ & $-136,67+(4,71$ Temperatura $)$ & 0,38 & 0,2461 \\
\hline
\end{tabular}

En el cuadro 3 se presentan valores de referencia reportados por diferentes autores en los períodos de preentrenamiento y postentrenamiento.

\section{DISCUSIÓN}

Las sesiones de entrenamiento requieren una minuciosa planificación y valoración de todos los factores que en él intervienen, ya que conforme la actividad física aumenta, también lo hace la actividad fisiológica de todos los sistemas corporales. Este incremento de la actividad física se vio reflejado en el presente estudio a través de los cambios en los valores de los diferentes indicadores metabólicos. En ambas razas la temperatura corporal incrementó después de realizar la rutina de ejercicios de Ring Francés. Este evento ha sido reportado previamente en estudios realizados por Rose y Bloomberg (1989) en Galgos y en Labradores Retrievers (Matwichuk y col 1999, Steiss y col 2004), donde mencionan que la temperatura rectal aumentó a $41{ }^{\circ} \mathrm{C}$ después de realizar ejercicio y se mantuvo elevada después de $30 \mathrm{~min}$ de finalizado el ejercicio.
Estos incrementos y posteriores descensos de temperatura observados en los experimentos podrían ser explicados por el aumento de la actividad metabólica que tienen que realizar los animales para desarrollar el esfuerzo muscular exigido por el trabajo mecánico, ya que una porción de energía de los nutrientes se convierte en calor durante el metabolismo celular (Forero y col 2006), por lo que cerca del $25 \%$ de la energía química se convierte en trabajo y el resto de la energía se convierte en calor (Hill 1998).

Van Citters y Franklin (1969) observaron en perros Alaska de trineo un aumento de la FC de 40-60 lat/min mientras dormían, y un incremento de hasta 80 a 100 lat/min cuando los perros despertaron y se mantuvieron en alerta; el incremento fue todavía superior cuando los perros se excitaron previo a la carrera (100 a $150 \mathrm{lat} / \mathrm{min})$ y durante de la carrera (250 a 300 lat $/ \mathrm{min}$ ). Este aumento de la frecuencia cardiaca es generado en respuesta al ejercicio como un mecanismo adaptativo (González y col 2008) que lleva a un aumento del gasto cardiaco para facilitar el aporte de sangre a los tejidos y así satisfacer el mayor requerimiento de oxígeno y demandas energéticas a los

Cuadro 3. Valores de referencia en diferentes períodos de entrenamiento.

Reference values in different periods of training.

\begin{tabular}{lccl}
\hline Variable & $\begin{array}{c}\text { Antes de la sesión } \\
\text { de entrenamiento }\end{array}$ & $\begin{array}{c}\text { Inmediatamente después de la } \\
\text { sesión de entrenamiento }\end{array}$ & Autores \\
\hline Lactato $(\mathrm{mg} / \mathrm{dL})$ & $21,297 \pm 6,936$ & $41,063 \pm 3,234$ & Rovira y col 2006; Rovira y col 2007 \\
$\mathrm{pH}$ & $7,391 \pm 0,023$ & $7,600 \pm 0,081$ & Matwichuk y col 1999; Steiss y col 2004 \\
$\mathrm{pCO}_{2}(\mathrm{mmHg})$ & $38,9 \pm 2,5$ & $13,9 \pm 2,2$ & Ilkiw y col 1989 \\
$\mathrm{pO}_{2}(\mathrm{mmHg})$ & $100,3 \pm 19,0$ & $130,8 \pm 14,0$ & Ilkiw y col 1989 \\
$\mathrm{Ca}^{++}(\mathrm{mmol} / \mathrm{L})$ & $2,6 \pm 0,3$ & $2,4 \pm 0,1$ & Angle y col 2009 \\
$\mathrm{K}^{+}(\mathrm{mmol} / \mathrm{L})$ & $4,7 \pm 0,4$ & $4,4 \pm 0,3$ & Angle y col 2009 \\
\hline
\end{tabular}


músculos activos. Por ejemplo, en perros Alaska el flujo en la aorta incrementa de 9 a 12 veces y el flujo coronario de 5 a 6 veces durante períodos de trabajo (Van Citters y Franklin 1969). En el presente estudio, la ausencia de variaciones en la FC en los animales de la raza P. Holandés durante las tres etapas de observación podría sugerir una mejor capacidad física y de adaptación durante el trabajo de Ring Francés, en comparación a la de los perros de la raza $P$. Belga Malinois, los cuales presentaron variaciones en la FC en las mismas etapas de muestreo. No obstante que en algunas ocasiones este aumento de la FC en perros de trabajo también puede ser generado como una respuesta anticipada al ejercicio (Forero y col 2006). Sin embargo, en nuestro estudio se evitó que se presentara esta situación de anticipación en ambos grupos.

Las respuestas del sistema circulatorio al ejercicio tienen como fin adaptar el flujo sanguíneo a los músculos activos (Olmos, 2010) dirigidas a aumentar el aporte de $\mathrm{O}_{2}$, tanto en músculo esquelético como al cardíaco, con el fin de sostener el aumento del metabolismo y facilitar la remoción de los productos metabólicos de desecho (García y col 1999). Al respecto, el incremento y disminución en las concentraciones de $\mathrm{pO}_{2}$ y $\mathrm{pCO}_{2}$, respectivamente, observados en este estudio en ambas razas como respuesta al ejercicio podrían deberse a un proceso de hiperventilación (jadeo) (Cunningham 2005) provocado como respuesta a la demanda física de $\mathrm{O}_{2}$ y a la termorregulación (Ilkiw y col 1989). Esta hiperventilación lleva consigo a un descenso en la $\mathrm{pCO}_{2}$ (hipocapnia) por aumento en su eliminación, y a su vez a un aumento en el $\mathrm{pO}_{2}$ por incremento en la entrada para este gas (Matwichuk y col 1999, Steiss y col 2004).

$\mathrm{El}$ aumento en las concentraciones de lactato se considera que es causado por la glicólisis anaerobia durante el ejercicio (Rovira y col $2007^{\mathrm{b}}$ ) y provee un indicador de adaptación y del grado de entrenamiento de un atleta humano o animal, debido a que refleja la dependencia de la vía anaeróbica como fuente de energía para realizar ejercicio muscular, donde aquellos menos entrenados muestran una mayor producción de lactato (García y col 1999). En nuestras observaciones, ambas razas presentaron un incremento significativo en las concentraciones de lactato posterior a la rutina de ejercicios, y consecutivamente una disminución. La ausencia de diferencias entre razas en estas dos etapas de observación sugiere una eficiencia de adaptación al ejercicio similar en las dos razas evaluadas. Asimismo, nuestros datos concuerdan con observaciones previas realizadas en Galgos (Pieschl y col 1992) y Labradores Retrievers (Steiss y col 2004) los cuales observaron aumentos significativos $(\mathrm{P}<0,05)$ en las concentraciones de lactato después del ejercicio (Rovira y col 2007a).

En nuestro estudio, el incremento observado en las concentraciones de lactato postentrenamiento difiere de estudios previos realizados en perros (Rose y Bloomberg 1989) y otras especies, en los cuales se ha observado que conforme incrementan los niveles de lactato, se presenta una disminución del $\mathrm{pH}$ sanguíneo posterior a un proceso de estrés (Herpin y col 1996, Trujillo y col 2007, Becerril y col 2009). Sin embargo, en nuestros resultados se observó un proceso de hiperlactatemia a la par de un incremento del $\mathrm{pH}$, resultados similares fueron reportados por Matwichuk y col (1999), lo cual podría ser debido al proceso de hiperventilación por el que cursaron los animales durante el ejercicio, ya que la hiperventilación lleva consigo a un descenso en el $\mathrm{pCO}_{2}$ (hipocapnia) por aumento en su eliminación y a su vez a un aumento en el $\mathrm{pO}_{2}$ (Rose y Bloomberg 1989) por incremento en la entrada para este gas. Esta disminución en la $\mathrm{pCO}_{2}$ incrementa el pH sanguíneo en los líquidos orgánicos (Guyton y Hall 2001, Heitz y Horne 2005, Forero y col 2006).

Durante el ejercicio físico, el metabolismo energético celular incrementa, ocurriendo cambios notables en las concentraciones de electrólitos y minerales en los diferentes compartimentos tisulares. De esta forma un aumento en las contracciones musculares conlleva a un incremento en los requerimientos de calcio y a una mayor utilización de las reservas de este mineral, y posteriormente una disminución en los niveles séricos (García y col 1999, Guyton y Hall 2001). Esto podría explicar la significativa disminución de calcio sanguíneo durante el período postentrenamiento observada en los perros de la raza P. Holandés de nuestro estudio. Asimismo, nuestros hallazgos coinciden con lo reportado con Burr y col (1997) y Rovira y col (2007a) con perros que realizaron ejercicios de Agility y perros de trineo, donde mencionan que las concentraciones de calcio disminuyeron significativamente después de realizar la actividad. Sin embargo, en la alcalosis aguda, como lo observado en los perros de este estudio, la disminución de la concentración de ion hidrógeno en plasma desplazan las formas de equilibrio del calcio (unido a proteínas, formando complejos con citrato o fosfato e ion calcio) hacia la derecha, aumentando la concentración de calcio unido a proteínas y complejado (con citrato o fosfato). El efecto inmediato es una disminución de la concentración de ion calcio en plasma (Fuentes y col 1998).

En conclusión, los cambios fisiometabólicos reportados en este estudio permitirán contar con antecedentes bioquímicos para poder evaluar la tolerancia al ejercicio y estimar el potencial de rendimiento competitivo en las dos razas de perros más empleadas en el Ring Francés. Estos resultados contribuyen en poder realizar y lograr una mejor selección de perros destinados para este fin basado en evidencias fisiológicas y metabólicas.

\section{RESUMEN}

En el presente estudio se valoró la respuesta fisiometabólica a una sesión de entrenamiento de Ring Francés en dos razas de perros de alto rendimiento. Se utilizaron 20 perros, machos y hembras de las razas Pastor Belga Malinois y Pastor Holandés entre 10 y 24 meses de edad con pesos entre 30 y $35 \mathrm{~kg}$ y que fueron sometidos durante 20 minutos a una rutina de Ring Francés. La toma de muestras se realizó en tres períodos: antes del entrenamiento, inmediatamente después y $1 \mathrm{~h}$ postentrenamiento. Las 
muestras sanguíneas se obtuvieron de la vena cefálica. Se determinaron los niveles de lactato, $\mathrm{pH}$, calcio, potasio y gases sanguíneos $\left(\mathrm{pCO}_{2}\right.$ y $\mathrm{pO}_{2}$ ) mediante un analizador de variables críticas sanguíneas. Se obtuvieron la frecuencia cardiaca y temperatura corporal. En ambas razas la temperatura, $\mathrm{pO}_{2}, \mathrm{pCO}_{2}$, lactato y $\mathrm{pH}$ incrementaron $(\mathrm{P}<0,05)$ después del período de entrenamiento, y disminuyeron $1 \mathrm{~h}$ después $(\mathrm{P}<0,05)$. La raza Pastor Belga Malinois mostró un incremento $(\mathrm{P}<0,05)$ en la Frecuencia Cardiaca en el postentrenamiento, y posteriormente una recuperación $(\mathrm{P}<0,05)$ hacia los valores de inicio. La raza $\mathrm{P}$. Holandés presentó una disminución $(\mathrm{P}<0,05)$ en los niveles de calcio posterior al entrenamiento, manteniéndose sin cambios en la recuperación. En conclusión, estos datos permitirán contar con antecedentes bioquímicos y fisiológicos para poder evaluar y estimar el potencial de rendimiento competitivo y lograr una mejor selección en las dos razas de perros más empleadas en el Ring Francés.

\section{AGRADECIMIENTOS}

El presente estudio es parte de los avances de la Tesis de Maestría de la primera autora, en el Programa de Posgrado en Ciencias de la Producción y de la Salud Animal de la Universidad Nacional Autónoma de México (UNAM). Se agradece de manera especial a Jorge Garrido, entrenador de las razas Pastor Belga Malinois y Pastor Holandés por su gran disposición y entrega para el experimento.

\section{REFERENCIAS}

Amtmann V, C Gallo, G van Schaik, N Tadich. 2006. Relaciones entre el manejo antemortem, variables sanguíneas indicadoras de estrés y $\mathrm{pH}$ de la canal en novillos. Arch Med Vet 38, 259-264.

Angle TC, JJ Wakshlag, RL Gillette, T Stokol, S Geske, TO Adkins, C Gregor. 2009. Hematologic, serum biochemical, and cortisol changes associated with anticipation of exercise and short duration high-intensity exercise in sled dogs. Vet Clin Pathol 38, 370-374.

Becerril HM, MS Alonso, CL Flores, IG Legarreta, AH Olmos, RN Ramírez, D Mota-Rojas. 2009. $\mathrm{CO}_{2}$ stunning may compromise swine welfare compared with electrical stunning. Meat Sci 81,233-237.

Becerril HM, MS Alonso, MEO Trujillo, IG Legarreta, RN Ramírez, PS Roldan, D Mota-Rojas. 2010. Changes in blood constituents of swine transported for 8 or $16 \mathrm{~h}$ to an Abattoir. Meat Sci 86, 945-948.

Brooks GA, GA Gaesser. 1980. End points of lactate and glucose metabolism after exhausting exercise. J Appl Physiol 49, 1057-1069.

Burr JR, GA Reinhart, RA Swenson, SE Swaim, DM Vaughn, DM Bradley. 1997. Serum biochemical values in sled dogs before and after competing in long-distance races. J Am Vet Med Assoc 211, 175-179.

Carter L, C Gallo. 2008. Effect of long distance transport by road and sea crossing on ferry on live weight losses and carcass characteristics in lambs. Arch Med Vet 40, 259-266.

Cunningham JG. 2005. Fisiología Veterinaria. $3^{\text {a }}$ ed. Elsevier, España.

Evans DL, CR Harris, DH Snow. 1993. Correlation of racing performance with blood lactate and heart rate after exercise in Thoroughbred horses. Equine Vet J 25, 441-445.

Forero LJH, APM Lozano, ROB Camargo. 2006. Parámetros fisiológicos en caninos pre y postcompetencia de agility en Bogotá, Colombia. Rev Med Vet 12, 57-71.

Fuentes AX, MJL Castiñeiras, JMC Queralto.1998. Bioquímica clínica y patología molecular. Vol II. 2 ed. Reverté, Barcelona, España.

García M, R Guzmán, I Cabezas, V Merino, C Palma, R Pérez. 1999. Evaluación del entrenamiento tradicional del caballo criollo chileno de rodeo mediante el análisis de variables fisiológicas y bioquímicas sanguíneas. Arch Med Vet 31, 167-176.

Gómez C, P Petron, M Andaur. 2004. Medición post-ejercicio de variables fisiológicas, hematológicas y bioquímicas en equinos de salto holsteiner. Rev Científica 14, 244-253.

González AJ, GC Crandall, JM Jonson. 2008. The cardiovascular challenge of exercising in the heat. $J$ Physiol 586, 45-53.
Grandjean D, JJP Vaissaire. 2003. Enciclopedia del perro. Aniwa Publishing, Royal Canin. Pp 436-449.

Guyton AC, JE Hall. 2001. Tratado de Fisiología Médica. McGraw Hill-Interamericana, México.

Heitz U, MM Horne. 2005. Fluidos, electrolitos y equilibrio ácido-base. $5^{\mathrm{a}}$ ed. Elsevier, España.

Herpin P, J Le Dividich, JC Hulin, M Fillaut, F de Marco, R Bertin. 1996. Effects of the level of asphyxia during delivery on viability at birth and early postnatal vitality of newborn pigs. J Anim Sci 74, 2067-2075.

Hill CR. 1998. The Nutritional Requirements of Exercising Dogs. $J$. Nutr 128, 2686S-2690S.

Ilkiw EJ, PE Davis, DB Church. 1989. Hematologic, biochemical, bloodgas, and acid-base values in Greyhounds before and after exercise. Am J Vet Res 50, 583-586.

Matwichuk CL, SM Taylor, CL Shmon, PH Kass, GD Shelton. 1999. Changes in rectal temperature and hematologic, biochemical, blood gas, and acid-base values in healthy Labrador Retrievers before and after strenuous exercise. Am J Vet Res 60, 88-92.

Mota-Rojas D, MH Becerril, LC Flores, PA Sánchez, ML González, AH Olmos, RN Ramírez, MS Alonso. 2006. Effects of mid-summer transport duration on pre- and post-slaughter performance and pork quality in Mexico. Meat Sci 73, 404-412.

Mota-Rojas D, MH Becerril, MEO Trujillo, MS Alonso, C Flores, IG Legarreta. 2009. Effects of pre-slaughter transport lairage and sex on pig chemical serologic profiles. J Anim Vet Advs 8, 246-250.

Olmos HA, MEO Trujillo, MS Alonso, RN Ramírez, D Mota-Rojas. 2008. Foetal monitoring, uterine dynamics and reproductive performance in spontaneous farrowing sows. J Appl Anim Res 33, 181-185.

Olmos HA, MEO Trujillo, MS Alonso, MH Becerril, RG Hernández, D Mota-Rojas. 2010. Porcine recombinant somatotropin administered to piglets during the first week of life: effects on metabolic and somatometric variables. Arch Med Vet 42, 93-99.

Olmos ZR. 2010. Sistema circulatorio, hemodinamia, circulaciones especiales y respuesta del sistema cardiovascular al ejercicio y la hemorragia. En: Caballero ChSC (ed). Fisiología veterinaria e introducción a la fisiología de los procesos productivos. FMVZUNAM, México, Pp 347-374.

Orozco-Gregorio H, DR Mota, MS Alonso, RN Ramírez, VYE Armenta, AN Ocampo, RG Hernández, MEO Trujillo, DG Villanueva. 2008. Short-term neurophysiologic consequences of intrapartum asphyxia in piglets born by spontaneous parturition. Int $\mathrm{J}$ Neurosci 118, 1299-1315.

Orozco-Gregorio H, DR Mota, HJ Bonilla, MEO Trujillo, MH Becerril, RG Hernández, DG Villanueva. 2010. Effects of administration of caffeine on metabolic variables in neonatal pigs with peripartum asphyxia. Am J Vet Res 71, 1214-1219.

Pieschl RL, PW Toll, DE Leith, LJ Peterson, MR Fedde. 1992. Acidbase changes in the running greyhound: contributing variables. $J$ Appl Physiol 73, 2297-2304.

Rose RJ, MS Bloomberg. 1989. Responses to sprint exercise in the greyhound: effects on haematology, serum biochemistry and muscle metabolites. Res Vet Sci 47, 212-218.

Rovira S, A Muñoz, M Benito.2007a . Fluid and electrolyte shifts during and after Agility competitions in dogs. J Vet Med Sci 69, 31-35.

Rovira S, A Muñoz, M Benito. 2007 . Hematologic and biochemical changes during canine agility competitions. Vet Clin Pathol 36, 30-35.

Sánchez AP, DR Mota, AO Nava, MEO Trujillo, ML González, ET Arch, AR Alfaro, RN Ramírez, MS Alonso. 2008. Effects of sildenafil on the fetal growth of guinea pigs and their capability to survive inducedintrapartum asphyxia. Am J Obstet Gynecol 198, 127.e1-127.e6.

Sánchez AP, DR Mota, MEO Trujillo, LAQ Zarco, MH Becerril, MS Alonso, AR Alfaro. 2009. Effect of prostaglandins for inducing birth, on weight, vitality and physiological response in newborn pigs. J Appl Anim Res 36, 113-118.

Steel GDR, HJ Torrie. 1986. Bioestadística, principios y procedimientos. McGraw-Hill, Bogotá, Colombia. 
Steiss J, HA Ahmad, P Cooper, C Ledford. 2004. Physiologic responses in healthy Labrador Retrievers during field trial training and competition. J Vet Inter Med 18, 147-51.

Tadich N, C Gallo, M Brito, D Broom. 2009. Effect of weaning and 48 hour transport by road and ferry on some blood indicators of welfare in lambs. Lives Sci 121,132-136.

Taylor LE, LP Ferrante, SD Kronfeld, TN Meacham.1995. Acid-base variables during incremental exercise in sprint-trained horses fed a high-fat diet. J Anim Sci 73, 2009-2018.

Taylor S, LC Shmon, DG Shelton, K Minor. 2008. Exercise-induced collapse of Labrador Retrievers: survey results and preliminary investigation of heritability. J Am Anim Hosp Assoc 44, 295-301.
Trujillo OME, DR Mota, AH Olmos, MS Alonso, ML González, HG Orozco, RN Ramírez, AO Nava. 2007. A study of piglets born by spontaneous parturition under uncontrolled conditions: Could this be a naturalistic model for the study of intrapartum asphyxia? Acta Biomed 78, 29-35.

Van Citters LR, DL Franklin. 1969. Cardiovascular performance of Alaska sled dogs during exercise. Circ Res 24, 33-42.

Warren LK, ML Lawrence, NK Thompson. 1999. The influence of betaine on untrained and trained horses exercising to fatigue. $J$ Anim Sci 77, 677-684.

Werner M, C Gallo. 2008. Effects of transport, lairage and stunning on the concentrations of some blood constituents in horses destined for slaughter. Lives Sci 115, 94-98. 